\title{
Front Matter: Volume 10463
}

, "Front Matter: Volume 10463," Proc. SPIE 10463, AOPC 2017: Space Optics and Earth Imaging and Space Navigation, 1046301 (12 December 2017); doi: $10.1117 / 12.2306549$

SPIE. Event: Applied Optics and Photonics China (AOPC2017), 2017, Beijing, China 


\title{
AOPC 2017: Space Optics and Earth Imaging and Space Navigation
}

\author{
Carl Nardell \\ Suijian Xue \\ Huaidong Yang \\ Editors
}

\section{4-6 June 2017 \\ Beijing China}

Sponsored by

SPIE

Chinese Society for Optical Engineering (China)

Organized by

Chinese Society for Optical Engineering (China)

Photoelectronic Technology Committee, Chinese Society of Astronautics (China)

Department of Cooperation and Coordination for Industry, Academe and Research, CHIA (China)

Science and Technology on Low-light-level Night Vision Laboratory (China)

Science and Technology on Electro-Optical Information Security Control Laboratory (China)

Published by

SPIE

\section{Volume 10463}


The papers in this volume were part of the technical conference cited on the cover and title page. Papers were selected and subject to review by the editors and conference program committee. Some conference presentations may not be available for publication. Additional papers and presentation recordings may be available online in the SPIE Digital Library at SPIEDigitalLibrary.org.

The papers reflect the work and thoughts of the authors and are published herein as submitted. The publisher is not responsible for the validity of the information or for any outcomes resulting from reliance thereon.

Please use the following format to cite material from these proceedings:

Author(s), "Title of Paper," in AOPC 2017: Space Optics and Earth Imaging and Space Navigation, edited by Carl Nardell, Suijian Xue, Huaidong Yang, Proceedings of SPIE Vol. 10463 (SPIE, Bellingham, WA, 2017) Seven-digit Article CID Number.

ISSN: 0277-786X

ISSN: 1996-756X (electronic)

ISBN: 9781510614079

ISBN: 9781510614086 (electronic)

Published by

SPIE

P.O. Box 10, Bellingham, Washington $98227-0010$ USA

Telephone +1 3606763290 (Pacific Time) · Fax +1 3606471445

SPIE.org

Copyright (C) 2017, Society of Photo-Optical Instrumentation Engineers.

Copying of material in this book for internal or personal use, or for the internal or personal use of specific clients, beyond the fair use provisions granted by the U.S. Copyright Law is authorized by SPIE subject to payment of copying fees. The Transactional Reporting Service base fee for this volume is $\$ 18.00$ per article (or portion thereof), which should be paid directly to the Copyright Clearance Center (CCC), 222 Rosewood Drive, Danvers, MA 01923. Payment may also be made electronically through CCC Online at copyright.com. Other copying for republication, resale, advertising or promotion, or any form of systematic or multiple reproduction of any material in this book is prohibited except with permission in writing from the publisher. The CCC fee code is $0277-$ $786 \mathrm{X} / 17 / \$ 18.00$.

Printed in the United States of America.

Publication of record for individual papers is online in the SPIE Digital Library.

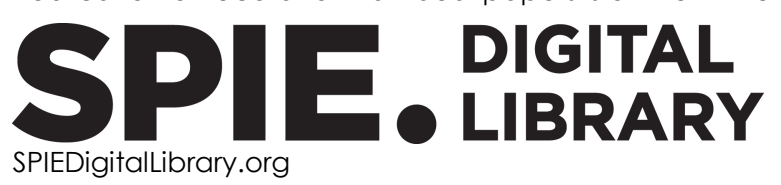

Paper Numbering: Proceedings of SPIE follow an e-First publication model. A unique citation identifier (CID) number is assigned to each article at the time of publication. Utilization of CIDs allows articles to be fully citable as soon as they are published online, and connects the same identifier to all online and print versions of the publication. SPIE uses a seven-digit CID article numbering system structured as follows:

- The first five digits correspond to the SPIE volume number.

- The last two digits indicate publication order within the volume using a Base 36 numbering system employing both numerals and letters. These two-number sets start with 00, 01, 02, 03, 04, 05, 06, 07, 08, 09, OA, OB ... 0Z, followed by 10-1Z, 20-2Z, etc. The CID Number appears on each page of the manuscript. 


\title{
Contents
}

\author{
vii Authors \\ xi Conference Committee \\ xiii Introduction
}

SPACE OPTICS AND EARTH IMAGING AND SPACE NAVIGATION

1046302 Interference and deception detection technology of satellite navigation based on deep learning [10463-32]

1046303 Calibration algorithm for center deviation of rotary tomography imaging [10463-18]

1046304 A heuristic constraint programmed planner for deep space exploration problems [10463-13]

1046305 A research on the positioning technology of vehicle navigation system from single source to "ASPN" [10463-4]

1046306 Visual navigation using natural landmark based on local features [10463-15]

1046307 Research on vehicle detection based on background feature analysis in SAR images [10463-29]

1046308 Enriching mission planning approach with state transition graph heuristics for deep space exploration [10463-31]

1046309 Decision-making method for small space aircraft navigation based on information fusion [10463-34]

10463 OA Spacial gyroscope calibration algorithm base on fusion filter [10463-3]

10463 OB Real-time single image dehazing based on dark channel prior theory and guided filtering [10463-59]

10463 OC Predicting the random drift of MEMS gyroscope based on K-means clustering and OLS RBF Neural Network [10463-22]

10463 OD Infrared nano-sensor based on doubly splited optomechanical cavity [10463-2]

10463 OE An innovative Neural-Fuzzy adaptive Kalman filter for ultra-tightly coupled GPS/INS integrated system [10463-14]

10463 OF Algorithm of GNSS positioning based on Doppler shift in incomplete condition of insufficient available satellites [10463-19] 
$104630 \mathrm{G}$ The application and development of ultra low expansion glass-ceramic in aerospace area [10463-20]

$10463 \mathrm{OH}$ A self-calibration method in single-axis rotational inertial navigation system with rotating mechanism [10463-12]

10463 Ol Simulation analysis of photometric data for attitude estimation of unresolved space objects [10463-25]

10463 0J Modeling amplitude SAR image with the Cauchy-Rayleigh mixture [10463-23]

10463 OK Real-time analysis for Stochastic errors of MEMS gyro [10463-17]

10463 OL A novel power control approach of multiple GNSS spoofing signals [10463-10]

10463 OM Research and development of a control system for multi axis cooperative motion based on PMAC [10463-9]

10463 ON Positioning navigation and timing service applications in cyber physical systems [10463-30]

1046300 The design of multi-core DSP parallel model based on message passing and multi-level pipeline [10463-7]

10463 OP A clock-aided positioning algorithm based on Kalman model of GNSS receiver clock bias [10463-8]

10463 OQ A new fault diagnosis algorithm for AUV cooperative localization system [10463-33]

10463 OR Gravity compensation in a Strapdown Inertial Navigation System to improve the attitude accuracy [10463-5]

10463 OS Simulation of some vital space radiation characteristics for a magnetosphere probing mission [10463-1]

10463 ОT Optical system design of CCD star sensor with large aperture and wide field of view [10463-16]

10463 OU Robust and intelligent algorithms for TDOA localization in distributed sensor networks [10463-21]

10463 OV Numerical simulation of optical synthetic aperture imaging system [10463-24]

10463 OW An intelligent anti-jamming network system of data link [10463-35]

10463 OX A BRDF statistical model applying to space target materials modeling [10463-36]

10463 OY Motion state analysis of space target based on optical cross section [10463-37]

$104630 Z$ Design of optical-mechanical structure for off-axis reflective star sensor ground calibration equipment [10463-38] 
1046310 Rearranging the lenslet array of the compact passive interference imaging system with high resolution [10463-39]

1046311 Miniaturized star tracker for micro spacecraft with high angular rate [10463-40]

1046312 Adaptive compressed sensing of remote-sensing imaging based on the sparsity prediction [10463-42]

1046313 Design and analysis of push-broom optical camera's following windows [10463-43]

1046314 Testing of an off-axis parabolic mirror based on hybrid compensation technology [10463-44]

1046315 Design of control system for piezoelectric deformable mirror based on fuzzy self-adaptive PID control [10463-45]

1046316 Adaptive optics correction based on stochastic parallel gradient descent technique using Zernike polynomials [10463-46]

1046317 Modal simulation and experimental verification of space-borne two dimensional turntable [10463-47]

1046318 Research on the method of improving the accuracy of CMM (coordinate measuring machine) testing aspheric surface [10463-48]

1046319 Research on convergence efficiency optimization of large and middle-scale silicon carbide mirrors [10463-50]

10463 1A Signal noise ratio analysis and on-orbit performance estimation of a solar occultation Fourier transform spectrometer [10463-51]

10463 1B Space-based detection of space debris by photometric and polarimetric characteristics [10463-58]

$104631 \mathrm{C}$ Optical system design of lunar rover navigation camera [10463-60]

10463 ID Variable curvature mirror having variable thickness: design and fabrication [10463-62]

$104631 \mathrm{E}$ High-precision processing and detection of the high-caliber off-axis aspheric mirror [10463-63]

$10463 \mathrm{lF}$ Thermal effects of optical antenna under the irradiation of laser [10463-64]

10463 IG High-resolution wavefront reconstruction using the frozen flow hypothesis [10463-65]

$10463 \mathrm{IH}$ Efficiency calibration of infrared single photon detectors by means of twin photons [10463-67]

1046311 Optomechanical integrated simulation of Mars medium resolution lens with large field of view [10463-68] 
$104631 \mathrm{~K}$ Temperature and nonlinearity correction methods for commercial CCD array spectrometers used in field [10463-70]

10463 IL Stray light and bandwidth corrections for commercial CCD array spectrometers [10463-71]

$104631 \mathrm{M}$ Dwell time method based on Richardson-Lucy algorithm [10463-72]

10463 iN A design of an on-orbit radiometric calibration device for high dynamic range infrared remote sensors [10463-73]

1046310 Lightweight structure design for supporting plate of primary mirror [10463-74]

10463 IP On advanced configuration enhance adaptive system optimization [10463-75]

10463 1Q Nonlinearity measurement of silicon detector and PMT detector [10463-76]

10463 IR The modeling and analysis of the image-rotation of the stabilizing mirror based on the spatial coordinate transformation [10463-77]

10463 is Design of high precision optical image stabilization system for high resolution Earth observation remote sensing [10463-79]

$104631 \mathrm{~T}$ Analysis and design of segment control system in segmented primary mirror (Invited Paper) [10463-80]

$104631 \mathrm{U}$ Lunar-edge based on-orbit modulation transfer function (MTF) measurement [10463-82]

$104631 \mathrm{~V}$ A new effective combined design method of optics and baffles for low obscuration ratio three-mirror system [10463-83]

10463 IW Influence of the platform jitter on intensity fluctuation for laser launch system [10463-84]

1046331 Frequency division multiplexed multi-color fluorescence microscope system [10463-800] 


\title{
Authors
}

Numbers in the index correspond to the last two digits of the seven-digit citation identifier (CID) article numbering system used in Proceedings of SPIE. The first five digits reflect the volume number. Base 36 numbering is employed for the last two digits and indicates the order of articles within the volume. Numbers start with 00, 01, 02, 03, 04, 05, 06, 07, 08, 09, OA, OB...0Z, followed by 10-1Z, 20-2Z, etc.

\author{
Ai, Jie, OD \\ Chen, Hongliang, 1P \\ Chen, Hongyue, 05 \\ Chen, Jianhua, OL \\ Chen, Junjie, OG \\ Chen, Mo, $1 T$ \\ Chen, Shuxin, OL \\ Chen, Su, 13 \\ Chen, Weiyi, 02 \\ Chen, Yu, 05 \\ Chen, Yuanpei, $\mathrm{OH}$ \\ Cheng, Ying, $1 \mathrm{U}$ \\ Chong, Xin, 12 \\ Cong, Wang, 18 \\ Cui, Kai, 17 \\ Cui, Pingyuan, 04, 08 \\ Dai, Caihong, 1K, 1L, 1Q \\ Dai, Chen, $1 \mathrm{E}$ \\ Deng, Pingke, 02 \\ Ding, Jiaoteng, 1D \\ Ding, Quanxin, 1P \\ Dong, Deng-feng, OM \\ Dong, Lijing, OG \\ Du, Hang, 19 \\ Du, Qingyu, 0J \\ Du, Xiaoping, 01 \\ Dun, Xiong, $1 \mathrm{~N}$ \\ Fan, Chengyu, IW \\ Fan, Xiangrui, OW \\ Fang, Jiaqi, OU \\ Feng, Yu, $1 \mathrm{H}$ \\ Gao, Shibo, 06 \\ Gao, Wei, 15 \\ Gong, Jie, 1D \\ Gou, Ruixin, Ol \\ Guo, Chunjie, IP \\ Guo, Jiu-yuan, OF \\ Guo, Kang, OA \\ Guo, Xiao-xiao, OM \\ Han, Ximeng, 14 \\ $\mathrm{He}$, Wenjing, $0 \mathrm{O}$ \\ He, Zhiyi, OU \\ Hou, Lizhou, 1A \\ Hu, Haibo, IS \\ $\mathrm{Hu}$, Heng, $\mathrm{OI}$ \\ $\mathrm{Hu}$, Jian, $0 \mathrm{O}$ \\ $\mathrm{Hu}$, Ruiguang, 06, 07 \\ Huang, Huang, $0 \mathrm{~J}$ \\ Huang, Sen, OL
}

Huang, Tong, IW

Jian, CUi, OU

Jiang, Aimin, is

Jiang, Bo, $1 \mathrm{M}$

Jiang, $\mathrm{Hu}, \mathrm{OS}$

Jiang, Lun, OT

Jiang, Xiao, 04

Jiang, Yongliang, 14

Jin, Guo Fan, 31

Jin, Hao, 08

Jin, Weiqi, $1 \mathrm{~N}$

Le, Vu Nam, 31

Li, Ang, 18, 1E

Li, Baosheng, 03

Li, Bicen, $1 \mathrm{~A}$

Li, Bin, $1 \mathrm{~T}$

Li, Chuanrong, 00

Li, Chunlei, OG

Li, Fu, IF

Li, Haizhou, 05

Li, Jianhua, 11

$\mathrm{Li}, \mathrm{Kui}, \mathrm{OH}$

Li, Ling, 1K, 1L, 1Q

Li, Shengyi, 19

Li, Tao, OD

Li, Xilong, 12

Li, Xu Peng, 10

Li, Yang, IV

Li, Yaping, 02

Li, Ying-chao, OT

Li, Zhi, OX, OY

Li, Zhifeng, 11

Li, Zhiguo, 17

Li, Zishen, OP

Liang, Yonghui, $1 \mathrm{G}$

Liao, Zhibo, IV

Lin, Jingyong, OW

Lin, Shangmin, 1C

Liu, Bei, 10

Liu, Chenghao, OX, OY

Liu, Dali, $1 \mathrm{~V}$

Liv, Dong, 14

Liv, Gang, 10

Liu, $\mathrm{Hao}, \mathrm{Ol}$

Liu, Hua, $1 \mathrm{P}$

Liv, Jiaqi, 09, 11

Liu, Jiarun, OW

Liu, Jie, $1 \mathrm{C}$

Liu, Jin, $1 G$ 
Liu, Meiying, 13, $1 \mathrm{C}$

Liu, Peng, 09

Liu, Shanlin, 03

Liu, Xinlong, $1 \mathrm{U}$

Liu, Xuewen, 1G

Liu, Yang, $1 \mathrm{C}$

Liv, Yang, $1 R$

Liu, Yunqing, $\mathrm{OZ}$

Liv, Zhaohui, 17

Liv, Zhuang, OT

Liu, Zhuowei, OL

Lu, Xiaoyun, 1B

Ma, Donglin, 14

Ma, Huimin, 16

Ma, Jingfang, OD

Ma, Liehua, OD

Ma, Zhen, $1 \mathrm{M}$

Meng, Fanrong, 00

Miao, Zhiyong, OK, OQ

Mu, Lei, 09

Niu, Jingyu, 00

Niu, Zhenhong, 11

Pan, Yue, 1B

Pang, Shuxia, 1B, 1C

Peng, Qiangqiang, 0J

Qiang, Li, 14

Qiao, Chunhong, IW

Qiao, Yan, 16

QU, Hemeng, 1 R

Qu, Yan Jun, 10

Qu, Yi, 02, ON

Shen, Chunshan, 16

Shen, Le, ID

Shen, Yang, 1B

Sheng, Yicheng, IN

Shi, Hongyang, $\mathrm{OK}, \mathrm{OQ}$

Song, $\mathrm{Ci}, 19$

Song, Zongxi, 10, 15

Sun, Qian, 05

Sun, $Y i, 1 F, 11$

Tang, Bo, 07

Tian, Qichen, OX, OY

Wang, Caiqin, 1A

Wang, Chao, OT

Wang, Feng, $1 \mathrm{C}$

Wang, Helong, $1 \mathrm{P}$

Wang, Hu, 13, 1B, 1C

Wang, Jun, OR

Wang, Lingcao, $\mathrm{OH}$

Wang, Lingguang, 13

Wang, Lingjie, IR

Wang, Wei, 10

Wang, Weidong, 09

Wang, Xiao, 10

Wang, Xingshu, OR

Wang, Yanfei, 1K, 1L, 1Q

Wang, Yang, 01

Wang, Yicheng, 03

Wang, Yipeng, OG

Wang, Yulei, 14
Wang, Zhen-yu, OC

Wang, Zhile, OV

Wen, Desheng, 10

Wu, Hongbo, $1 \mathrm{R}$

WU, Jiabao, OE

Wu, Xiaojing, ON

WU, Zhifeng, 1K, 1L, 1Q

Xian, Hao, $1 T$

Xiang, Yanjun, OD

Xiao, Liping, 06, 07

Xiao, Nan, 15

Xiao, $\mathrm{Si}, 1 \mathrm{~N}$

Xie, Xiaopeng, 1D

$X U, C a n, O X, O Y$

$X u$, Fan, OA

$X U$, Guangzhou, 11

$X U$, Jieping, $1 G$

$\mathrm{XU}$, Liang, 1D

$X U$, Lingdi, 18, $1 \mathrm{E}$

$X U$, Pengmei, $1 \mathrm{~A}$

$X \cup, R u i, 04,08$

$X \cup$, Weicai, 14

$\mathrm{X}$, Wenming, 08

Xue, Yaoke, 13, 1C

Yan, Lisong, 14

Yang, Huai Dong, 31

Yang, Jianfeng, 1F, 11

Yang, Senlin, 12

Yang, Shuai, OR

Yang, Wenqiang, 1F, 11

Yang, Yongying, 14

Yao, Yinwei, OJ

Yi, Hongwei, $1 \mathrm{U}$

You, Taihua, OA

Yu, Guangwei, OG

Yu, Jinpei, OS

Yu, Wenhao, $1 T$

Yuan, Hong, OP

Yue, Xiang, 03

Yun, Hongquan, 07

Zeng, Lingchuan, ON

Zhang, Bochuan, 07

Zhang, Cong, 07

Zhang, Jianping, $1 \mathrm{R}$

Zhang, Jing, 05

Zhang, Jinghui, IW

Zhang, Jiqing, OG

Zhang, Li-jie, OC

Zhang, Pengfei, IW

Zhang, Shuqing, OV

Zhang, Si Chun, 31

Zhang, Tingcheng, $1 \mathrm{~V}$

Zhang, Weiyi, 06

Zhang, Wenying, $\mathrm{OZ}$

Zhang, Xiaoguang, 02

Zhang, Xiao-lin, OF

Zhang, Xibin, $1 \mathrm{C}$

Zhang, Xin Rong, 31

Zhang, Xin, IR

Zhang, Yangyang, $1 \mathrm{H}$ 
Zhang, Yeping, OD

Zhang, Yi, OK, OQ

Zhang, Yingjie, $1 \mathrm{E}$

Zhang, Yongqiang, 17

Zhang, Yuanyuan, OG

Zhang, Zan, OB

Zhao, Hui, 1D

Zhao, Pengyu, $1 \mathrm{H}$

Zhou, Chenghao, OV

Zhou, Chunmei, ow

Zhou, Feng, iN

Zhou, Junhong, $1 \mathrm{Q}$

Zhou, Liang, 17

Zhou, Liwei, IP

Zhou, Wei-hu, OM

Zhu, Haoran, $\mathrm{OZ}$

Zhu, Jing, $O R$

Zhu, Lingyao, OP

Zhu, Shengying, 08

Zou, Dinghua, 17

Proc. of SPIE Vol. $104631046301-9$

Downloaded From: https://www.spiedigitallibrary.org/conference-proceedings-of-spie on 26 Apr 2023 Terms of Use: https://www.spiedigitallibrary.org/terms-of-use 
Proc. of SPIE Vol. 10463 1046301-10 Downloaded From: https://www.spiedigitallibrary.org/conference-proceedings-of-spie on 26 Apr 2023
Terms of Use: https://www.spiedigitallibrary.org/terms-of-use 


\title{
Conference Committees
}

\author{
Conference Chairs
}

Guangjun Zhang, Beihang University (China)

Byoungho Lee, Seoul National University (Korea, Republic of)

\section{Conference Committee}

Desheng Jiang, Wuhan University of Technology (China)

Hequan Wu, Chinese Academy of Engineering (China)

Jianquan Yao, Tianjin University (China)

Jianwei Pan, University of Science and Technology of China (China)

Junhao Chu, Shanghai Institute of Technical Physics, CAS (China)

Junen Yao, Beihang University (China)

Lijun Wang, Changchun Institute of Optics, Fine Mechanics and Physics, CAS (China)

Lin Li, The University of Manchester (United Kingdom)

Liwei Zhou, Beijing Institute of Technology (China)

Min Gu, RMIT University (Australia)

Shibin Jiang, AdValue Photonics Inc. (United States)

Toyohiko Yatagai, Utsunomiya University (Japan)

Wei Wang, Beijing Institute of Aerospace Control Devices, CASC (China)

Weidou Ni, Tsinghua University (China)

Zuyan Xu, Technical Institute of Physics \& Chemistry, CAS (China)

Program Committee

Anand Krishna Asundi, Nanyang Technological University (Singapore)

Bing Zhao, Jilin University (China)

Byoungho Lee, Seoul National University (Korea, Republic of)

Carl Nardell, Google Skybox Imaging (United States)

Chunhua Shen, The University of Adelaide (Australia)

Haimei Gong, Shanghai Institute of Technical Physics, CAS (China)

Honghai Liu, University of Portsmouth (United Kingdom)

Huaidong Yang, Tsinghua University (China)

Huijie Zhao, Beihang University (China)

Jannick Rolland, Institute of Optics, University of Rochester (United States)

Jin Lu, Tianjin Jinhang Institute of Technical Physics (China)

Jin Yu, Université Claude Bernard Lyon 1 (France)

Jinxue Wang, SPIE

Lijun Wang, Changchun Institute of Optics, Fine Mechanics and Physics, CAS (China)

Lin Li, The University of Manchester (United Kingdom)

Lan Jiang, Tsinghua University (China) 
Long Zhang, Shanghai Institute of Optics and Fine Mechanics, CAS (China)

Mengxia Xie, Beijing Normal University (China)

Min Gu, RMIT University (Australia)

Min Qiu, Zhejiang University (China)

Shibin Jiang, AdValue Photonics Inc. (United States)

Suijian Xue, National Astronomical Observatories, CAS (China)

Tsutomu Shimura, The University of Tokyo (Japan)

Wei Hang, Xiamen University (China)

Wei Wang, Beijing Institute of Aerospace Control Devices, CASC (China)

Weibiao Chen, Shanghai Institute of Optics and Fine Mechanics, CAS

(China)

Wolfgang Osten, Universität Stuttgart (Germany)

Xiandeng Hou, Sichuan University (China)

Xiangping Li, Jinan University (China)

Xiaocong Yuan, Shenzhen University (China)

Xiaodi Tan, Beijing Institute of Technology (China)

Yadong Jiang, University of Electronic Science and Technology of China (China)

Yanbiao Liao, Tsinghua University (China)

Yong Bi, Academy of Opto-Electronics, CAS (China)

Yongtian Wang, Beijing Institute of Technology (China)

Zhe Wang, Tsinghua University (China)

Zhiping Zhou, Peking University (China)

Session Chairs

1 Carl Nardell, Google Terra Bella (United States)

2 Larry Gordley, Global Atmospheric Technologies and Sciences (United States)

3 Yang Huaidong, Tsinghua University (China)

4 Hao Lei, Shanghai Astronomical Observatory, CAS(China) 


\section{Introduction}

Applied Optics and Photonics China (AOPC2017) is the annual conference of the CSOE, and one of the largest academic and industry activities in the field of optical and optoelectronic technology in China. The organization committee has built a platform of academic exchanges, industry exhibitions, and cooperation negotiations in one. There are 8 technical conferences, 7 themes of the Exhibition and approximately 600 technical presentations. We sincerely hope that the research and development of optoelectronic technology are promoted, and the international cooperation between industry and the optical and optoelectronic fields are enhanced.

AOPC2017 is technically co-sponsored by the Chinese Society for Optical Engineering, the Optical Society of Korea (OSK), Optics and Photonics Society of Singapore (OPSS), European Optical Society (EOS), Optical Society of Japan (OSJ) and SPIE. There are also 60 cooperative organizers to support the conference. We received over 1209 contributions from more than 15 countries, including the United States, the United Kingdom, Germany, France, Spain, Australia, Canada, Mexico, Brazil, Japan, Korea, Thailand, Singapore, the Russian Federation, China, and more. There are more than 700 presentations published in the Proceedings of SPIE. After careful discussion, we suggested four keynote speeches which are presented by famous scientists from Germany, Australia, Japan and China. 138 excellent invited talks were presented, 45 are from outside of China. Their presentations reflect first-class research in the field of optics and photonics technology. On behalf of the Organization Committee of AOPC, I express thanks to all the invited speakers and authors for their contributions and support of the conference.

Finally, on behalf of Prof. Zhuang Songlin, and other co-chairmen, and the Organization Committee of AOPC, I would like to heartily thank our sponsors and cooperating organizers for all they have done for the conference, the participants and friends for their interests and efforts in helping us to make the conference a success, the program committee for their effective work and valuable advice, and especially the AOPC2017 Secretariat and the staff of SPIE for their tireless effort and outstanding services in preparing the conference and publishing the Proceedings.

We wish AOPC2017 great success! Hope to see you next year!

Guofan Jin 
Proc. of SPIE Vol. 10463 1046301-14 Downloaded From: https://www.spiedigitallibrary.org/conference-proceedings-of-spie on 26 Apr 2023
Terms of Use: https://www.spiedigitallibrary.org/terms-of-use 